\title{
Could PD-1/PDL1 immune checkpoints be linked to HLA signature?
}

\author{
Pierpaolo Correale1, Rita E Saladino², Valerio Nardone³, Rocco Giannicola1, Rita Agostino, \\ Luigi Pirtoli $^{4}$, Michele Caraglia*,5 (iD), Ciro Botta ${ }^{6}$ \& Pierosandro Tagliaferri ${ }^{7}$ \\ ${ }^{1}$ Medical Oncology Unit, Grand Metropolitan Hospital "Bianchi-Melacrino-Morelli", Reggio Calabria, Italy \\ ${ }^{2}$ Tissue Typing Unit, Grand Metropolitan Hospital "Bianchi-Melacrino-Morelli", Reggio Calabria, Italy \\ ${ }^{3}$ Radiotherapy Unit, "Ospedale del Mare", Naples, Italy \\ ${ }^{4}$ Department of Biology, College of Science \& Technology, Temple University, Philadelphia, PA, USA \\ ${ }^{5}$ Department of Precision Medicine, University of Campania "L. Vanvitelli", Via L. De Crecchio, 780138 Naples, Italy \\ ${ }^{6}$ Hematology Unit, Annunziata Hospital, Cosenza, Italy \\ ${ }^{7}$ Medical Oncology Unit, AUO "MaterDomini" \& "Magna Graecia" University, Catanzaro, Italy \\ *Author for correspondence: Tel.. +39 081566 5871; Fax: +39 081566 5863; michele.caraglia@unicampania.it
}

"In summary, we believe that the correlation between treatment response to immune-checkpoint inhibitors and the occurrence of irAEs and HLA signature in cancer patients deserves further investigation in large perspective studies."

First draft submitted: 9 September 2019; Accepted for publication: 31 October 2019; Published online: 9 December 2019

Keywords: HLA alleles • irAEs • outcome • PD-1/PDL-1-blockade

The outstanding clinical expansion of monoclonal antibodies (mAbs) to programmed cell death receptor-1 (PD1) (nivolumab and pembrolizumab) and PD-1 ligand-1 (PDL-1) (atezolizumab, avelumab and durvalumab) has received an increasing level of interest regarding immunotherapy and multidrug combinations, for the treatment of a number of common human malignancies [1]. Some patients treated with these agents receive remarkable benefits in term of quality of life, progression-free (PFS) and overall survival (OS) [2,3]. However, a significant percentage of these patients experience immune-related adverse events (irAEs), while others present with an ultra-rapid disease progression, defined as hyperprogression. Research in to the mechanisms related to these events is an active field of investigation worldwide, whose results are expected to provide new insights to design new combinations, to identify potentially responsive patients and to prevent irAEs' occurrence [4].

\section{Mechanisms behind mAb immune-checkpoint blockade}

Immune-checkpoint blocking $\mathrm{mAbs}$ are unable to exert a direct antitumor effect alone, rather they rescue tumorinfiltrating cytotoxic-T-lymphocytes (CTLs) inactivated throughout the PD-1 pathway due to interaction with PDL-1/2 molecules expressed on inflammatory and cancer cells [1]. Clinical efficacy of these mAbs is therefore indirectly dependent on the presence of a sufficient number of tumor-reactive CTLs that are able to trigger proficient tumor rejections. Tumor-infiltrating CTLs restored by PD-1/PDL1 blockade represent a state of immune response to antigenic proteins expressed by tumor cells, such as tumor-associated antigens (TAAs) and tumor-specific antigens (TSAs). Thus, their involvement in carcinogenesis largely precedes the immune-oncological treatment [5]. PD-1/PDL1 blockade, similar to the active specific immunotherapy, requires the presence of CTL $\left(\mathrm{CD} 3^{+} \mathrm{CD} 8^{+}\right)$ clones expressing T-cell receptors (TCRs) that are able to recognize tumor antigen-derived epitope peptides bound to class I human leukocyte antigen (HLA) molecules, on either cancer cells or antigen presenting cells (APCs) [5]. Specifically, TCRs on CTLs bind 9-10 amino acid peptides bound to homologous class I HLA alleles on both target cells and APCs [5-9]. Other T-cell subsets $\left(\mathrm{CD}^{+} \mathrm{CD}^{+}\right)$, with the ability to enhance ( $\mathrm{T}$ helpers; $\left.\mathrm{T}_{\mathrm{h}}\right)$, redirect $\left(\mathrm{Th}_{1}\right.$, $\mathrm{Th}_{2}, \mathrm{Th}_{17}$, etc.) or attenuate ( $\mathrm{T}$ regulators; $\mathrm{T}_{\text {reg }} \mathrm{s}$ ) the immune response, express a different TCR, which instead binds larger antigen-derived peptides bound to homologous class II HLA molecules on APC and inflammatory cell lineages only [5-9]. The aforementioned epitope peptides, available for either class I or II HLA, derive from the intracellular proteolysis of protein antigens occurring in all of the eukaryotic cells. Subsequently, epitope peptide

Future $\because \cdots$ Medicine 
binding to different HLA isotypes takes place in the endoplasmic reticulum and is restricted by the expression of allele-specific amino acid consensus motifs [5-9]. In this light, tumor cells process and present their own peptides for CTL recognition, while APCs and inflammatory cells uptake and process antigens released by tumor tissues in the blood stream and lymphatic drainage. This is a result of spontaneous death, hypoxia, radiotherapy, specific treatments or immune-related killing [10] in a process known as cross priming. These APCs then present the epitope peptides to $\mathrm{T}$ cells together with secondary co-accessory molecule-mediated messages that, in turn, trigger $\mathrm{T}$ - and B-cell clone proliferation. Consequently, this may be responsible for both maintenance of the antitumor immune response and also for the occurrence of irAEs when the immune response is redirected to self-antigens [9].

\section{Existing \& supposed biomarkers predictive of response to immunological checkpoint inhibitors}

At present, the most relevant research fields concerning clinical and immune-biological elements predictive of response to PD-1/PDL1 blockade and autoimmunity are mainly focused on PDL-1 expression, as well as inflammatory infiltrate in tumor tissue, histology, gender, tumor mutation burden as a source of antigens, peripheral blood cytokine profiling and intra-tumor expression of specific immune-cell subsets. When considering the research of potential mechanisms for acquired immune resistance, researchers are evaluating T-cell exhaustion and co-existing additional immune checkpoints, along with mAb-related depletion of T cells due to anti-PD-1 mAbs being the most active CTL clones [11]. Considering their critical role in conditioning T-cell immune-priming and CTL-mediated tumor cell killing, it is reasonable to believe that differences in class I and II HLA haplotype could have an important role in conditioning either outcome and/or irAEs in patients receiving PD-1/PDL-1 blocking mABs. There is clear evidence that tumor cells that acquire a homozygosis status for class I HLA by deleting one of the two parental allele genes become partially invisible. Therefore, they become refractory to PD-1 rescued immune effectors since they present a much lower number of epitopes available for CTL recognition [12]. Class I HLA upregulation induced by exposure to either gamma-interferon, cytotoxic drugs or ionizing radiation was able to enhance tumor cell susceptibility to specific CTL clones, which supports this hypothesis $[13,14]$.

In this context, the study of CTL epitope peptide binding to HLA molecules has been considered a critical point for the development of vaccine strategies and anti-cancer active-specific immunotherapy over the last 30 years. Allele-specific amino acid consensus motifs have been largely described in the literature and categorized by Parker et al. and Rammensee et al. They created network algorithms able to identify the presence of epitope sequences and their potential binding affinity for each specific HLA allele, just by scanning the primary structure of known viral and tumor-derived protein antigens $[15,16]$. Both algorithms have allowed the identification of many HLA allele-specific immunogenic peptides. These have been tested in preclinical models including class I specific peptide vaccines from various TAAs including the carcino-embryonic antigen, human telomerase reverse transcriptase, prostate-specific antigen, parathyroid hormone-related peptide, thymidylate synthase poly-epitope peptide, EGFR, gp170, melan-A and many others [16].

\section{Mechanisms at the basis of HLA haplotypes involvement in the prediction of response}

The literature suggests that each protein antigen present in its primary structure includes one or more amino acidic sequences (epitopes) potentially able to bind different class I/II HLA isotypes. It is clear that both the number of epitopes and their relative binding affinity is completely different for each different HLA allele [17]. It is consequential that patients with different HLA profiles also have a completely different family of epitopes, which are available for immune-cell recognition and CTL-mediated killing, even in the presence of the same antigens. Therefore, we can speculate that different outcomes and frequency of irAEs in patients subjected to the same immune-checkpoint blockade, are largely influenced by a pre-existing immune competence and reactivity strongly related to the different amount of high affinity epitope peptides bound to class I/II HLA molecules. This could be dramatically different on the basis of the divergent HLA haplotype. We believe that different HLA haplotypes may influence the amount of efficient CTL precursors available for a direct antitumor response, long-term memory and continuous immune response maintenance, which are able to affect patients' survival. A different HLA haplotype is also correlated to a divergent susceptibility to autoimmunity, as demonstrated by the fact that specific alleles in class I and class II HLA have been already correlated to a high risk of rheumatoid arthritis and other auto-immune disorders [18]. Our group has recently shown that the survival of pretreated metastatic non-small-cell lung cancer patients receiving nivolumab was directly correlated to the occurrence of both irAEs and a treatment-related increase in extractable nuclear antigen, antinuclear antibodies and anti-smooth muscle antibodies [19]. This effect was explained as part of 
an antigen cascade or antigen migration process, as recorded in other immunotherapy, chemo-immunotherapy and radio-immunotherapy models. More recently, we also found that the outcome, as well as the occurrence of irAEs of the same patients, also correlated to the expression of specific HLA allelic groups like $A^{*} 01, B^{*} 51, D R B 1^{*} 03$, $D R B 1^{*} 11$, etc.

\section{Conclusion}

We believe that the identification of lung cancer patients with a weak immune-reactive HLA haplotype could gain significant benefit by performing future combination studies where immune-checkpoint inhibitors are combined with anti-cancer vaccines. The latter are, in fact, able to trigger a T-cell immune response to cryptic antigens and epitopes, for which no clonal deletion or exhaustion occurs.

Additionally, the identification of hyper-immune-reactive HLA haplotypes may receive great attention in the future considering the clinical development of additional PD-1/PDL-1 immune-checkpoint blockers in combination with platinum doublets, anti-angiogenic drugs and/or radiotherapy as a front-line regimen in non-small-cell lung cancer patients. This development has been consequential to the promising results of rapid fast-track trials. Nevertheless, recent studies have found that the level of risk for immune-related deaths within the first 60 days of the treatment is very high due to massive immune-system overstimulation [20].

In summary, we believe that the correlation between treatment response to immune-checkpoint inhibitors and the occurrence of irAEs and HLA signature in cancer patients deserves further investigation in large perspective studies. Moreover, an accurate and real-world evidence-based multimodal definition of tumor immunogenicity at baseline, as well as HLA haplotype characterization, can be of help to define not only responsive patients but also patients with high risk of lethal irAEs.

\section{Financial \& competing interests disclosure}

The authors have no relevant affiliations or financial involvement with any organization or entity with a financial interest in or financial conflict with the subject matter or materials discussed in the manuscript. This includes employment, consultancies, honoraria, stock ownership or options, expert testimony, grants or patents received or pending, or royalties.

No writing assistance was utilized in the production of this manuscript.

\section{References}

1. Pardoll DM. The blockade of immune checkpoints in cancer immunotherapy. Nat. Rev. Cancer 12(4), 252-264 (2012).

2. Creelan BC. Update on immune checkpoint inhibitors in lung cancer. Cancer Control. 21(1), 80 (2014).

3. Horn L, Spigel DR, Vokes EE et al. Nivolumab versus docetaxel in previously treated patients with advanced non-small-cell lung cancer: two-year outcomes from two randomized, open-label, Phase III trials (CheckMate 017 and CheckMate 057). J. Clin. Oncol. 35(35), 3924-3933 (2017).

4. Nardone V, Pastina P, Giannicola R et al. How to increase the efficacy of immunotherapy in NSCLC and HNSCC: role of radiation therapy, chemotherapy, and other strategies. Front. Immunol. 9, 2941 (2018).

5. Wolfel T, Klehmann E, Muller C, Schutt KH, Meyer zum Buschenfelde KH, Knuth A. Lysis of human melanoma cells by autologous cytolytic T cell clones. Identification of human histocompatibility leukocyte antigen A2 as a restriction element for three different antigens. J. Exp. Med. 170(3), 797-810 (1989).

6. Crowley NJ, Darrow TL, Quinn-Allen MA, Seigler HF. MHC-restricted recognition of autologous melanoma by tumor-specific cytotoxic T cells. Evidence for restriction by a dominant HLA-A allele. J. Immunol. 146(5), 1692-1699 (1991).

7. Falk K, Rotzschke O, Stevanovic S, Jung G, Rammensee HG. Allele-specific motifs revealed by sequencing of self-peptides eluted from MHC molecules. Nature 351(6324), 290-296 (1991).

8. Hunt DF, Henderson RA, Shabanowitz J et al. Characterization of peptides bound to the class I MHC molecule HLA-A2.1 by mass spectrometry. Science 255(5049), 1261-1263 (1992).

9. McDonnell AM, Robinson BW, Currie AJ. Tumor antigen cross-presentation and the dendritic cell: where it all begins? Clin. Dev. Immunol. 2010, 539519 (2010).

10. Sanchez-Paulete AR, Teijeira A, Cueto FJ et al. Antigen cross-presentation and T-cell cross-priming in cancer immunology and immunotherapy. Ann. Oncol. 28(Suppl. 12), xii44-xii55 (2017).

11. Sharma P, Hu-Lieskovan S, Wargo JA, Ribas A. Primary, adaptive, and acquired resistance to cancer immunotherapy. Cell 168(4), 707-723 (2017).

12. Gettinger S, Choi J, Hastings K et al. Impaired HLA class I antigen processing and presentation as a mechanism of acquired resistance to immune checkpoint inhibitors in lung cancer. Cancer Discov. 7(12), 1420-1435 (2017). 
13. Correale P, Botta C, Cusi MG et al. Cetuximab +/- chemotherapy enhances dendritic cell-mediated phagocytosis of colon cancer cells and ignites a highly efficient colon cancer antigen-specific cytotoxic T-cell response in vitro. Int. J. Cancer 130(7), 1577-1589 (2012).

14. Gallegos CE, Michelin S, Dubner D, Carosella ED. Immunomodulation of classical and non-classical HLA molecules by ionizing radiation. Cell. Immunol. 303, 16-23 (2016).

15. Parker KC, Bednarek MA, Coligan JE. Scheme for ranking potential HLA-A2 binding peptides based on independent binding of individual peptide side-chains. J. Immunol. 152(1), 163-175 (1994).

16. Rammensee H, Bachmann J, Emmerich NP, Bachor OA, Stevanovic S. SYFPEITHI: database for MHC ligands and peptide motifs. Immunogenetics 50(3-4), 213-219 (1999).

17. Paul S, Weiskopf D, Angelo MA, Sidney J, Peters B, Sette A. HLA class I alleles are associated with peptide-binding repertoires of different size, affinity, and immunogenicity. J. Immunol. 191(12), 5831-5839 (2013).

18. Gregersen PK, Silver J, Winchester RJ. The shared epitope hypothesis. An approach to understanding the molecular genetics of susceptibility to rheumatoid arthritis. Arthritis Rheum. 30(11), 1205-1213 (1987).

19. Giannicola R, D'Arrigo G, Botta $C$ et al. Early blood rise in auto-antibodies to nuclear and smooth muscle antigens is predictive of prolonged survival and autoimmunity in metastatic-non-small cell lung cancer patients treated with PD-1 immune-check point blockade by nivolumab. Mol. Clin. Oncol. 11(1), 81-90 (2019).

20. Mulkey F, By K, Theoret MR et al. Analysis of early mortality in randomized clinical trials evaluating anti-PD-1/PD-L1 antibodies: a systematic analysis by the United States Food and Drug Administration (FDA). J. Clin. Oncol. 2019 ASCO Annual Meeting, Chicago, IL, USA (2019). 